\title{
Langage de Coordination Multi-Agent Sécurisé
}

\author{
Mahdi Zargayouna* , _ Flavien Balbo** \\ * Université Paris-Est, IFSTTAR, GRETTIA \\ 2 av. de la Butte Verte \\ 93166 Noisy-Le-Grand, France \\ hamza-mahdi.zargayouna@ifsttar.fr \\ ** Université Paris Dauphine, Laboratoire CNRS-Lamsade \\ Place du Maréchal de Lattre de Tassigny \\ 75775 Paris Cedex 16, France \\ balbo@lamsade.dauphine.fr
}

\begin{abstract}
RÉSUMÉ. Cet article traite des questions de sécurité des systèmes multi-agents dans les langages de coordination orientés données. Ces langages se fondent sur un espace de données partagées par les agents et utilisées pour coordonner leurs activités. Nous étendons ces langages avec un mécanisme de sécurité fondé sur des conditions associées à toute donnée de l'espace partagé. Ces conditions sont définies avec une granularité fine et sont fondées sur les états des agents. Ceci est rendu possible grâce à deux idées principales : la considération au niveau du langage d'une abstraction des états des agents sous la forme de données et l'introduction d'un mécanisme d'interaction plus riche que les templates de l'état de l'art. Ce mécanisme de sécurité permet autant aux agents qu'au concepteur d'interdire les interactions indésirables.
\end{abstract}

ABSTRACT. This paper discusses the security issues of multiagent systems in data driven coordination languages. These languages rely on a data space shared by the agents and used to coordinate their activities. We extend these languages with a main distinguishing feature, which is the possibility to define fine-grained state-based security conditions, associated with every datum in the shared space. Two main ideas makes it possible: the consideration at language level of an abstraction of agents' states in the form of data and the introduction of a richer interaction mechanism than state-of-the-art templates. This novel security mechanism allows both agents and designers to prohibit undesirable interactions.

MOTS-CLÉS : Langages de coordination, Interaction, Systèmes Multi-Agents, Sécurité

KEYWORDS: Coordination languages, Interaction, Multiagent Systems, Security

$1^{\text {re }}$ soumission à $R S T I-R I A$, le 31 janvier 2012 


\section{Introduction}

L'expansion constante des réseaux d'ordinateurs et le développement continu d'applications distribuées oriente la conception des systèmes logiciels modernes à se concentrer de plus en plus sur la réutilisation et l'intégration de composants logiciels. Ainsi, nous avons assisté à un passage des applications autonomes à des réseaux distribués en interaction, qui appellent à des méthodologies et des outils bien définis, visant à intégrer des composants logiciels hétérogènes. L'approche des systèmes multi-agents (SMA) partage ces mêmes objectifs d'intégration d'applications autonomes. Dans un SMA, le contrôle est distribué sur un réseau d'agents autonomes, et le fonctionnement du système émerge des comportements locaux des agents le composant (Sycara, 1998). Cependant, il est difficile de développer de tels systèmes, sans connaître la structure globale du système, ni les agents qui y évolueront. De tels systèmes sont dits ouverts, dans le sens où les agents peuvent rejoindre et quitter le système librement (Ciancarini, 1990).

Les langages de coordination orientées données, avec le langage pionnier Linda (Carriero et al., 1986), offrent un moyen simple et flexible de concevoir des systèmes ouverts. Linda consiste en un ensemble de primitives sur un espace de données appelé tuplespace, pouvant être théoriquement ajoutées à n'importe quel langage de programmation. Outre la création de processus, les primitives permettent d'ajouter, lire et récupérer (lire et supprimer) des données de l'espace partagé. L'accès aux données du tuplespace est associatif i.e. par contenu (grâce à des templates). Linda et les extensions qui en en été faites forment désormais la famille des modèles de coordination orientés données, en opposition aux modèles de coordination orientés contrôle (Papadopoulos et al., 1998). Ces derniers définissent des systèmes dans lesquels les processus communiquent avec leur environnement avec des interfaces bien définies, généralement appelées des «ports d'entrée » et «ports de sortie». Les relations producteur-consommateur sont formées par des canaux entre les ports de sortie des producteurs et les ports d'entrée des consommateurs. Ces canaux sont point-àpoint, ou forment parfois une relation un-à-plusieurs, dans le cadre d'une diffusion restreinte.

Néanmoins, lorsqu'ils sont utilisés dans des environnements non sécurisés, les systèmes adhérant à un modèle de coordination orienté données se retrouvent face à des menaces directement liées à ce principe de partage des informations. En effet, l'une des exigences vitales lors de la conception de systèmes ouverts est la sécurité. Afin de garantir quelques propriétés relatives à la sécurité, différentes méthodes fondées sur la cryptographie ont été proposées. Elles sont appliquées à un large éventail d'applications, garantissant la confidentialité des messages échangés, leur authentification, leur signature numérique, etc. Les protocoles de sécurité adoptés sont typiquement fondés sur une topologie à base de canaux (Focardi et al., 2006). Les auteurs dans (Pfleeger et al., 2002) classent les menaces à la sécurité en : i) menace à la confidentialité; ii) menace à l'intégrité ; iii) menace à la disponibilité, iv) menace à l'authenticité. Dans la littérature, plusieurs modifications ont été apportées aux langages de type Linda pour faire face aux menaces de sécurité. Dans (Focardi et al., 2006), les auteurs classent les 
langages orientés données sécurisés en langages orientés entités et en langages orientés connaissances. L'idée derrière l'approche orientée entités est que chaque ressource liste les agents autorisés à y accéder. Dans le cas de l'approche orientée connaissances, les tuples, l'espace de données ou les simples champs de données sont enrichis avec des informations et seuls les agents prouvant leur connaissance de ces informations peuvent accéder à ces ressources.

Nous désirons équiper les langages de coordination orientés données avec un mécanisme de sécurité à grain fin, permettant la protection différenciée de chaque donnée échangée, afin de permettre la mise en oeuvre de ces langages dans la conception des SMA. La sécurité à grain fin permet d'associer des règles de sécurité, non pas à un espace de données partagé, ni à un ensemble de champs, mais à chaque donnée élémentaire échangée. Notre proposition peut être classée comme une approche orientée entités, mais au lieu de lister les agents qui peuvent accéder à une donnée, nous proposons un langage dans lequel ces agents sont décrits «symboliquement », c'est à dire que leurs propriétés sont définies sans les désigner nommément. En effet, en raison de cette exigence de liste nominative, les auteurs dans (Focardi et al., 2006) avancent que l'approche orientée connaissances est plus adaptée pour les systèmes ouverts. En ce sens, nous proposons une approche orientée entités adaptée pour les systèmes ouverts. Nous donnons aux agents la possibilité de spécifier, quand ils ajoutent une donnée à l'espace partagé, les conditions dans lesquelles elle peut être lue ou récupérée par d'autres. De plus, le concepteur spécifie les conditions générales dans lesquelles les agents peuvent ou ne peuvent pas ajouter une certaine donnée à l'espace, suivant la logique de l'application. A cette fin, nous apportons plusieurs modifications au modèle originel Linda, et proposons un nouveau langage appelé LACIOS (Language for Agent Contextual Interaction in Open Systems), qui est la matérialisation linguistique du modèle modifié.

La définition des entités coordonnées dans LACiOs diffère des langages de coordination de la littérature. D'abord, les agents dans LACIOS ont un état qu'ils peuvent utiliser dans la définition de leurs situations d'interaction. Par exemple, un agent peut décider de ne recevoir des requêtes que s'il est libre, i.e. n'effectuant aucune autre action. Ensuite, les agents LACIOS ont un comportement défini par une composition de processus, notamment parallèle. Ainsi, un agent ne se bloque pas nécessairement dans l'attente des informations recherchées, mais peut activer un comportement à la recherche de cette information alors que d'autres continuent leur traitement. Enfin, les agents dans LACIOS ont une mémoire locale indépendante de l'environnement dans laquelle ils stockent les informations échangées avec les autres.

Notre objectif à terme est de proposer un langage totalement multi-agent, dans le sens où les agents poursuivent des objectifs définis d'une manière déclarative et avec un déploiement du système totalement distribué. Notre idée dans le travail proposé ici est de définir un langage, fondé sur les travaux antérieurs en langages de coordination, qui soit assez riche pour ces développements futurs. Nous croyons que LACIOS, de par sa richesse relative et les aspects relatifs à la sécurité, est un bon candidat pour ces développements. Le langage LACIOS s'adresse aux concepteurs de SMA ouverts 
désirant faire interagir leurs agents via un espace partagé, sans encourir les risques de sécurité inhérents au partage des informations échangées.

Afin de présenter notre proposition nous suivrons le plan suivant. Dans la section 2 , nous motivons notre proposition et donnons une vue d'ensemble de LACIOS. Dans la section 3 nous donnons les fondamentaux de la syntaxe du langage. Dans la section 4, nous définissons notre mécanisme de gestion de la sécurité. Dans la section 5 , nous présentons le langage de programmation JAVA-LACIOS qui est l'implementation en Java du langage de coordination LACIOS. Nous discutons le positionnement de notre proposition vis à vis de l'état de l'art dans la section 6 avant de conclure et de proposer quelques pistes de recherche futures.

\section{Motivation et vue d'ensemble}

Afin d'illustrer les concepts introduits par le langage LACIOS, nous commençons par introduire un scénario d'agents voyageurs dans une gare. Ce scenario sera évoqué tout au long de cet article afin de motiver l'introduction de nouveaux concepts et pour illustrer la syntaxe et la sémantique du langage. Il s'agit d'une gare où des humains, des services de planification, de réservation, de paiement, etc. et des sources d'information coexistent. Ces différents acteurs doivent partager des informations concernant les départs, les arrivées, les retards etc. Tous les voyageurs désirant utiliser les services à l'intérieur de la gare disposent d'un moyen de communication, tel qu'un téléphone portable avec un navigateur, un PDA ou un ordinateur portable avec une technologie d'accès à distance (3G, Wi-Fi, etc.). Tous les voyageurs et services de la gare disposent d'agents les représentant dans le SMA.

\subsection{Motivation}

Dans notre scenario, les agents interagissent par échange de données dans un espace partagé à la manière des modèles de coordination orientés données. Les menaces à la sécurité que nous considérons sont : i) menace à l'authenticité; ii) menace à la confidentialité ; iii) menace à la disponibilité ; iv) menace à l'intégrité.

Une menace sur l'authenticité survient quand un agent agit comme s'il s'agissait d'un autre. Considérons un agent voyageur qui ajoute un message à l'espace de données partagées, prétendant qu'il émane d'un agent train et donnant une information concernant un retard. Cette information peut induire en erreur les autres agents voyageurs, qui rateraient leur train s'ils ont cette information et agissent en conséquence. Pour traiter la menace à l'authenticité, un processus d'authentification externe est généralement utilisé, avec un agent désigné ou un processus système qui traite les requêtes d'authentification avec un protocole particulier. Le client présente les données nécessaires à son authentification au processus d'authentification et, s'il est authentifié, il reçoit un jeton d'authentification. Il fournit ce jeton au système lors de son interaction avec l'espace partagé. Le jeton correspond à un ensemble de droits d'accès, 
ou il peut agir comme clé de cryptage, utilisée pour décrypter les messages ultérieurs. Dans (Bryce et al., 2001), les auteurs fournissent une approche cryptographique pour les protocoles d'authentification. Cependant, si cette procédure est toujours nécessaire pour interagir avec un espace partagé dans les systèmes ouverts, rien n'empêche l'agent, une fois accepté, d'usurper l'identité d'un autre. Dans LACiOs, une vérification de l'authenticité est effectuée à chaque fois qu'un agent ajoute une donnée à l'espace partagé, et le mécanisme proposé va au delà de l'authenticité puisque certaines insertions de données, indépendantes de l'identification de l'agent, peuvent être également interdites, si le concepteur estime qu'elles corrompent la cohérence du système.

Les menaces à la confidentialité sont relatives à l'interception par un agent d'une information ou d'un message destiné à un autre. Il s'agit d'une menace à la disponibilité si l'agent supprime le message ou l'information. Par exemple, considérons un agent voyageur $v$ qui essaie de lire ou de prendre un message émis par un agent train pour un autre agent voyageur, avec une information sur le prix du billet qu'il lui propose. L'agent $v$ doit être empêché de lire (confidentialité) et de prendre (disponibilité) ce message. Pour ce faire, plusieurs langages reposent sur des espaces privés (e.g. (De Nicola et al., 1998)), dont le créateur peut déterminer les droits d'accès des autres agents à ses données. Une autre approche est d'associer des clés avec les données insérées (e.g. (Vitek et al., 2003)); un agent doit avoir la clé correspondante pour pouvoir accéder à ces données. Cependant, ces approches bien qu'utilisant le modèle de l'espace partagé pour la communication entre agents, en utilisent un autre pour protéger les données. Par conséquent, elles tendent à altérer le modèle originel (par exemple, le calcul de SecOS est fondé sur le $\pi$-calculus asynchrone (Vitek et al., 2003), exploitant sa communication à base de canaux). Dans LACIOS, les données sont protégées à la Linda, i.e. associativement, en utilisant une sorte de template pour décrire qui peut et qui ne peut pas accéder une certaine donnée.

Enfin, les menaces sur l'intégrité sont relatives à la modification d'un message ou d'une information d'un agent par d'autres. Dans les langages orientés données, aucun agent ne peut modifier les données partagées. L'unique action possible est de prendre une donnée et d'en créer une nouvelle avec des informations différentes. Si la récupération d'un objet est gardée par un mécanisme de disponibilité, il n'y a pas besoin d'un mécanisme spécifique pour les menaces à l'intégrité dans les langages orientés données, y compris LACIOS.

\subsection{Vue d'ensemble de LACIOS}

Un SMA écrit en LACios est défini par un ensemble dynamique d'agents locaux interagissant avec un environnement, qui est composé d'un ensemble dynamique d'objets (figure 1). Puisque les agents dans LACIOS n'interagissent pas directement, mais via l'environnement, notre définition d'un agent est proche de la définition générale de (Wooldridge et al., 1995) : 
Définition 1 (Agent) Un agent est un système informatique qui est situé dans un environnement et qui est capable d'appliquer des actions autonomes dans le but de satisfaire ses buts.

Un SMA écrit en LACIOS est un système ouvert selon deux dimensions. Premièrement, comme pour tout langage orienté données, les agents dans LACIOS peuvent rejoindre et quitter le système librement. Dans ce cas, un agent local représente l'agent externe dans l'environnement afin d'y exécuter une partie des instructions de l'agent distant (e.g. agent $_{b}$ est représenté par agent $_{2}$ dans la figure 1). L'agent externe est un agent qui n'accède pas directement à l'environnement, et qui crée un agent en lui fournissant les instructions à exécuter. Deuxièmement, des systèmes externes - non modélisés - et des utilisateurs peuvent interagir avec le SMA. Ces systèmes externes (e.g. les trains, les voyageurs) peuvent exécuter des agent locaux qui interagissent avec l'environnement SMA (e.g. agent $_{3}$ et agent $_{4}$ dans la figure 1). Un agent local peut également être indépendant (e.g. agent ${ }_{1}$ dans la figure 1) exécutant ses propres instructions. Ainsi, le SMA modélisé s'exécute sur un hôte particulier, où des agents (locaux) ajoutent, lisent et prennent des objets (données) de/à l'environnement SMA selon une modélisation homogène des différents composants du système réel. Chaque agent est soit indépendant, soit représentant un système/utilisateur non modélisé dans le SMA.

Les agents qui sont définis dans un programme LACIOS sont les agents «locaux ». Les utilisateurs, les agents externes et les systèmes externes qui sont représentés par un agent dans le SMA ne sont pas modélisés, seules leurs actions sont observées dans le SMA, à travers le comportement non-déterministe de l'agent local. Les agents distants (comme agent $_{b}$ ) fournissent leur nouvel état à l'agent local à chaque fois qu'il change.

Du point de vue de la sécurité, nous considérons que les agents locaux créés sur le serveur sont issus d'un mécanisme sécurisé assurant leur authenticité. Par conséquent, LACIOS doit assurer la sécurité au sein de l'environnement, et poursuit deux objectifs : i) permettre un contrôle global par l'environnement des insertions d'objets par les agents, pour s'assurer que les nouveaux objets ne sont pas frauduleux (authenticité et disponibilité), ii) permettre un contrôle local par les agents qui peuvent spécifier qui peut accéder à l'objet qu'ils ajoutent à l'environnement pour s'assurer de son caractère privé (authenticité, confidentialité, disponibilité et intégrité). Pour ce faire, les agents doivent avoir un état définissant ce qu'ils sont. Il s'agit là de la première modification que nous effectuons sur le modèle originel Linda : la considération au niveau du langage d'une abstraction des états des agents sous forme de données. Ces états sont définis comme un ensemble de couples propriété $\leftarrow$ valeur (e.g. \{identifiant $\leftarrow 10$, position $\leftarrow$ "Quai $\left.{ }_{2} "\right\}$ ). Les états des agents dans LACIOS sont des données représentant l'état des agents qui sont utilisées par l'environnement pour effectuer des appariements (ils ne sont pas directement accessibles par les autres agents). La deuxième modification est la proposition d'un mécanisme d'interaction riche, utilisant des opérateurs et des variables, qui permettent de définir le mécanisme de gestion de la sécurité. Les sections suivantes sont dédiées à la spécification du langage et à la description du mécanisme de sécurité. 


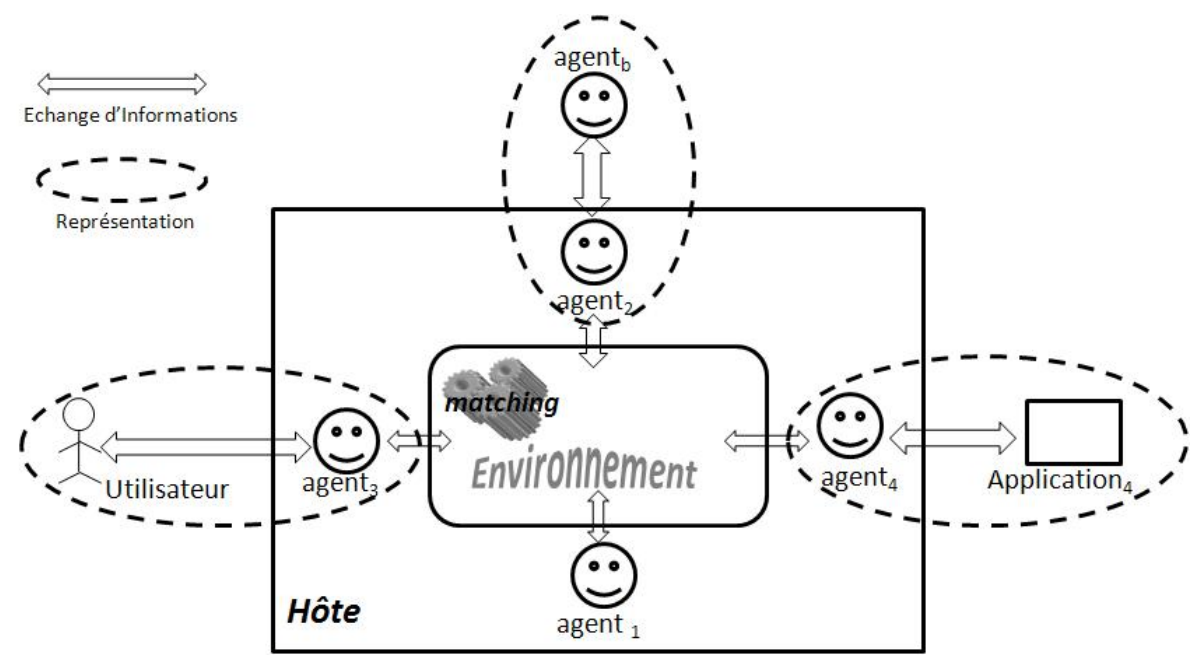

Figure 1. Architecture de LACIOS

\section{Syntaxe basique et sémantique informelle pour L ACIOS}

LACIOS est un langage orienté données pour la conception et l'implémentation de SMA ouverts et sécurisés. Pour la spécification des comportements des agents, nous utilisons quatre primitives inspirées par Linda et un ensemble d'opérateurs empruntés du CCS de Milner (Milner, 1989). Un SMA écrit en LACiOs est défini par un ensemble dynamique d'agents interagissant avec un environnement - dénoté $\Omega_{E N V}$, qui est composé d'un ensemble d'objets. La figure 2 illustre le principe général de LACIOS. Les agents sont définis par un comportement (un processus), un état (données) et une mémoire locale dans laquelle ils stockent les objets qu'ils perçoivent ou récupèrent depuis l'environnement. Les agents peuvent percevoir (lire seulement) et/ou récupérer (lire et prendre) des objets depuis l'environnement. D'abord, nous présentons les quatre primitives de LACIOS; leurs paramètres vont être définis en même temps que les détails du langage.

$$
\mu::=\text { spawn } \mid \text { add } \mid \text { update } \mid \text { look }
$$

La primitive spawn lance un nouvel agent et lui donne un état initial et un comportement. Une action add ajoute un objet à l'environnement. La primitive update change les anciennes valeurs de l'état de l'agent vers de nouvelles définies en paramètre.

Différemment des primitives traditionnelles d'accès à l'environnement, la primitive look permet aux agents de percevoir et de récupérer des objets en même temps. Elle permet de lire un objet, le récupérer ou les deux à la fois. Cette primitive recherche 
des objets dans l'environnement qui satisfont les contraintes spécifiées par l'agent. Les agents peuvent utiliser leurs propres états dans l'expression paramètre d'un look, qui est accessible par l'environnement seulement, quand l'expression paramètre est évaluée. Notons que les autres agents ne peuvent accéder directement à l'état de l'agent à travers des expressions look. Pour être observable par les autres, un agent doit ajouter un objet le représentant à l'environnement, d'une manière autonome, comme il peut ne publier qu'une partie de son état comme dans la figure 2. Avoir des données qui représentent les agents dans l'environnement permet aux agents du système de se découvrir les uns les autres en interrogeant simplement l'environnement à la manière de Linda.

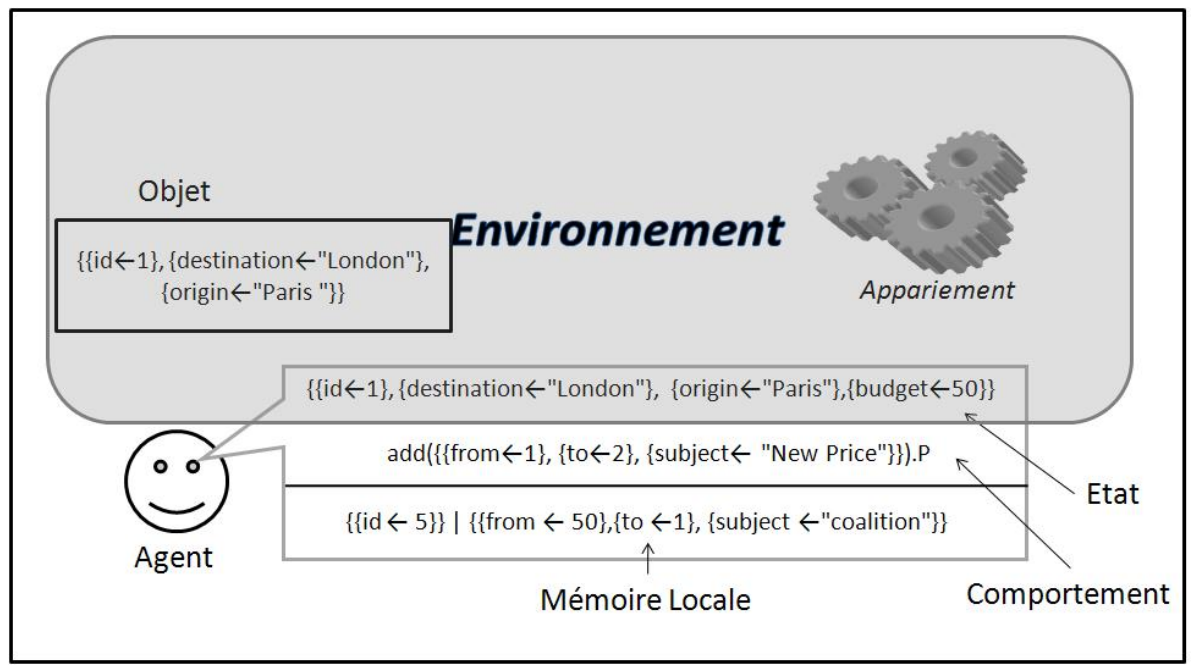

Figure 2. Schéma Général

\subsection{Structure de données}

La structure de n-uplets sur laquelle se fondent les modèles de coordination de type Linda ne nous satisfait pas, car elle dispose seulement de deux critères afin de discriminer un n-uplet : l'ordre des champs et leur type. La structure de données que nous retenons est celle d'un système d'information standard, où les données sont représentées par un ensemble de couples propriété-valeur. En effet, lorsqu'on modélise un système d'information (e.g. (Komorowski et al., 1998)), on s'appuie généralement sur cette représentation (un enregistrement dans une table par exemple).

Considérons un agent voyageur. Un objet représentant cet agent pourrait être décrit ainsi : 


$$
\{i d \leftarrow \text { "v1", age } \leftarrow 25, \text { fumeur } \leftarrow \text { vrai, budget } \leftarrow 120\}
$$

Chaque donnée échangée dans le système est décrite par une «description», i.e. un ensemble de couples propriété $\leftarrow$ valeur, et toutes les propriétés dans le langage sont typées.

Définition 2 (Types) Les types du langage sont définis comme type ${ }_{1}, \ldots$, type $_{n b t}$. Chaque type $_{i}$ est un ensemble tel que $\forall(i, j) \in\{1, \ldots, n b t\}^{2}, i \neq j$, type $_{i} \cap$ type $_{j}=$ $\{$ nil $\}$

Notation 1 Nous définissons l'ensemble des valeurs supportées par le langage comme $\mathcal{T}=\bigcup_{i=1}^{\text {nbt }}$ type $_{i}$.

REMARQUE. -

Nous supposons l'existence du type booléen dans le langage, i.e. $\exists i \in\{1, \ldots, n b t\}$, type $_{i}=\left\{\right.$ vrai $_{\text {, faux }, \text { nil }\}}$

La valeur particulière nil a un double emploi dans notre syntaxe. D'une part, elle représente toute erreur sémantique du langage. Lors de l'évaluation d'une expression syntaxiquement valide, si elle génère une erreur sémantique, elle est évaluée à nil. D'autre part, elle représente l'absence d'une propriété. En effet, une propriété dont l'évaluation est nil est considérée comme inexistante. Les propriétés sont utilisées afin de décrire chaque agent et chaque objet du SMA, et chaque propriété est définie par son type.

Définition 3 (Propriétés) $\mathcal{N}$ est l'espace de propriétés, il s'agit d'un ensemble dénombrable de propriétés. Une propriété $\pi \in \mathcal{N}$ est définie par un type type $(\pi) \in$ $\left\{\right.$ type $_{1}, \ldots$, type $\left._{n b t}\right\}$.

Notation 2 Nous notons unknown $n_{\pi}$ une valeur de type type $(\pi)$ mais dont la valeur est inconnue. Par exemple unknown $n_{\text {age }}$ est une propriété de type type $($ age $)=\mathbb{N}$, mais dont la valeur est (temporairement) inconnue.

Une description est une collection de propriétés avec leurs valeurs correspondantes.

Définition 4 (Descriptions) DS est l'ensemble des descriptions. Une description est une fonction faisant correspondre des propriétés à des valeurs, i.e. $d \equiv\left\{\pi \leftarrow v_{\pi} \mid\right.$ $\left.v_{\pi} \in \operatorname{type}(\pi)\right\}_{\pi \in \mathcal{N}}$. La correspondance est omise quand $v_{\pi}=$ nil. Nous utilisons $d(\pi)$ afin d'accéder à la valeur $v_{\pi}$. Pour chaque description, l'ensemble de propriétés $\{\pi \mid d(\pi) \neq$ nil $\}$ est fini. 
Ainsi donc, une propriété dont la valeur est nil désigne une propriété non définie pour cet agent ou objet, i.e. qui n'existe pas (e.g. la propriété rayon pour un rectangle). Une description représente un ensemble fini de couples propriété-valeur tel que valeur est différente de nil.

Définition 5 (Entités) $\Omega=\mathcal{A} \cup \mathcal{O}$ est l'ensemble des entités $d u$ SMA, avec $\mathcal{A}$ l'ensemble d'agents et $\mathcal{O}$ l'ensemble d'objets. Chaque entité $\omega$ a une description comme décrite plus haut notée $d_{\omega}$. La valeur de la propriété $\pi$ de l'entité $\omega$ est notée $d_{\omega}(\pi)$.

Prenons l'exemple des voyageurs dans une gare. Une entité (voyageur) $v_{1}$ peut avoir la description suivante :

$$
\begin{gathered}
d_{v_{1}} \equiv\{i d \leftarrow \text { "v } 1 ", \text { catégorie } \leftarrow \text { "voyageur", fumeur } \leftarrow \text { vrai, budget } \leftarrow 120, \\
\text { destination } \left.\leftarrow \text { "Rennes", train } \leftarrow \text { unknown } n_{\text {train }}\right\}
\end{gathered}
$$

Une entité train $t$ peut avoir la description suivante :

$d_{t_{1}} \equiv\left\{i d \leftarrow\right.$ "t $t_{1}$ ", catégorie $\leftarrow$ "train", capacité $\leftarrow 125$, destination $\leftarrow$ "Rennes", prix $_{\text {ind }} \leftarrow 80$, prix $\left._{\text {groupe }} \leftarrow 50\right\}$,

avec prix $x_{\text {ind }}$ désignant le prix pour une seule personne, et prix $x_{\text {groupe }}$ désignant le prix pour un groupe de personnes. Par convention, un groupe est formé d'au moins trois personnes.

Les types définis pour cet exemple ne sont pas détaillés car intuitifs, par exemple, type $($ fumeur $)=$ booléen, type $($ age $)=\mathbb{N}_{+}^{*} \cup$ nil, etc.

REMARQUE. -

Nous supposons l'existence du type référence dans le langage, une variable de type référence désigne une entité dans $\Omega$, i.e. $\exists i \in\{1, \ldots, n b t\}$, type $e_{i}=\Omega \cup\{$ nil $\}$.

Nous considérons notre proposition, en termes de structure de données, comme un compromis entre les n-uplets Linda et les autres structures de données proposées dans la littérature depuis Linda (objets pour Javaspaces (Freeman et al., 1999), enregistrements relationnels pour TSpaces (Wyckoff et al., 1998), prédicats pour TucSon (Omicini et al., 1999)). Les couples propriété-valeur sont un peu plus expressifs que les n-uplets (nommage, typage et relaxation de l'ordre des champs) tout en restant assez généraux pour être codés en objets, en prédicats ou en enregistrements dans une table relationnelle.

\subsection{Expressions}

Les expressions sont construites avec des valeurs, des propriétés et des opérateurs. Elles sont utilisées par les agents pour décrire les données qu'ils gèrent, soit localement, soit pour interagir avec l'environnement.

Définition 6 (Opérateurs) Chaque opérateur op du langage est défini par : 
- (i) arité $(o p)$ Le nombre de paramètres de l'opérateur,

- (ii) $\operatorname{par}(o p):\{1, \ldots$, arité $(o p)\} \rightarrow\{1, \ldots, n b t\}, \operatorname{par}(o p)(i)$ donne l'indice du type du $i$ ème paramètre de l'opérateur op,

- (iii) $\operatorname{ret}(o p) \in\{1, \ldots, n b t\}$ donne l'indice du type de la valeur résultant de l'évaluation de op.

Par exemple, soit type $_{1}=$ booléen $\cup\{$ nil $\}$. L'opérateur et est défini comme suit : $\operatorname{arité}($ et $)=2, \operatorname{par}($ et $)(1)=\operatorname{par}($ et $)(2)=1$ et $\operatorname{ret}($ et $)=1$. Outres les opérateurs basiques, des opérateurs additionnels peuvent être définis par le programmeur, spécifiant un processus complexe de l'agent.

Une expression peut être simplement une valeur, mais aussi un opérateur ou une propriété. Par exemple, destination $\neq$ "Paris" est une expression (booléenne). Si une expression est une propriété, elle se réfère à une propriété de l'agent qui est en train de l'évaluer. Par exemple, quand la propriété destination est utilisée dans le comportement d'un agent $a$, elle désigne la destination de $a$. Si une propriété compagnon de l'agent $a$ est de type référence, compagnon.destination désigne la destination du compagnon de $a$.

Définition 7 (Expressions) Exp est l'ensemble des expressions. Une expression e $\epsilon$ Exp est générée via la grammaire du tableau 1.

\begin{tabular}{ll}
\hline$e::=$ nil &, avec $v \in \mathcal{T} \backslash$ nil \\
$\mid v$ &, avec $\pi \in \mathcal{N}$ \\
$\mid \pi$ &, avec op un opérateur du langage, \\
$\mid$ op $(e, \ldots, e)$ & et nil n'apparaît dans aucun $e$ \\
$\mid \pi . e$ &, avec $\pi \in \mathcal{N}$ et type $(\pi)=\Omega$ \\
\hline
\end{tabular}

Tableau 1. Syntaxe d'une expression

Comme décrit plus haut, une description est un ensemble de couples propriétévaleur. Désormais, nous pouvons associer à chaque propriété, non plus une valeur, mais une expression. Le résultat est une «description symbolique ». Une description symbolique est transformée en une description lors de l'évaluation des expressions la composant.

Définition 8 (Descriptions symboliques) SDS est l'ensemble des descriptions symboliques. Une description symbolique est une description qui fait correspondre des propriétés $\pi$ à des expressions $e_{\pi}$, i.e. $s d s \equiv\left\{\pi \leftarrow e_{\pi} \mid \text { type }\left(e_{\pi}\right)=\text { type }(\pi)\right\}_{\pi \in \mathcal{N}}$. 
Ci-après la définition des primitives add et update, avec leurs paramètres (descriptions symboliques).

$$
\mu::=\cdots \mid \text { add }(s d s) \mid \text { update }(s d s)
$$

Pour ajouter un objet, un agent exécute $a d d(s d s)$, et un objet dont la description est l'évaluation de $s d s$ est ajouté à $\Omega_{E N V}$. Par exemple, add $\left(\right.$ ticket $_{i d} \leftarrow$ ticket.id,prix $\leftarrow$ ticket.prix +20 , propriétaire $\leftarrow i d\})$ insère un objet dans l'environnement dont le propriétaire est égal à l'id de l'agent appelant, ticket $_{i d}$ est égal à la propriété $i d$ d'un objet dans la mémoire de l'agent appelant (pointée par la propriété de type référence ticket) et dont le prix est de 20 supérieur au prix payé par l'agent. La primitive update (sds) met à jour les propriétés de l'agent avec l'évaluation des expressions dans $s d s$. Quand update(sds) est exécuté, la valeur de chaque propriété $\pi$ dans $s d s$ devient égale à l'évaluation de l'expression correspondante $e_{\pi}$. Par exemple, si un agent $a$ exécute update $(\{$ budget $\leftarrow$ budget -20 , destination $\leftarrow$ "Budapest" $\}$ ), son budget est décrémenté de 20 et sa destination devient "Budapest".

\subsection{Appariement}

Puisque nous considérons une structure de données qui est plus riche que les nuplets, nous utilisons également un mécanisme d'appariement qui est plus riche que les templates. Pour ce faire, la syntaxe des expressions est enrichie avec des variables, qui désignent des objets inconnus de l'agent, mais qui vont être découverts durant le processus d'appariement et vont être remplacés par des objets de l'environnement avant leur évaluation. Ci-après la définition d'une variable.

Définition 9 (Variables) $\mathcal{X}$ est un ensemble (potentiellement infini) de variables. Une variable $x \in \mathcal{X}$ est définie par son type type $(x) \in\left\{\right.$ type $_{1}, \ldots$, type $\left._{n b t}\right\}$.

Par exemple, considérons l'expression booléenne $e:$ t.destination $=$ "London" $\wedge$ t.prix $\leq$ budget. Dans cette expression, $t$ désigne un objet, inconnu pour le moment, où la propriété destination de $t$ doit être égale à "London" et le prix doit être inférieur au budget de l'agent pour que l'expression soit évaluée à vrai. Quand l'expression est évaluée à vrai, l'agent exécutant look avec $e$ comme paramètre va percevoir ou récupérer l'objet.

Nous pouvons désormais fournir la définition complète de la primitive look.

$$
\mu::=\cdots \mid \operatorname{look}\left(s d s_{p}, s d s_{r}, e\right)
$$

Nous choisissons d'utiliser une seule primitive pour accéder à l'environnement. La primitive $l o o k\left(s d s_{p}, s d s_{r}, e\right)$, avec $s d s_{p}$ et $s d s_{r}$ des descriptions symboliques, 
permet la perception et la récupération (perception et suppression de $\Omega_{E N V}$ ) simultanées d'objets. Elle bloque jusqu'à ce qu'un ensemble d'objets $C$ devient présent dans $\Omega_{E N V}$, tel que l'expression $e$ est évaluée à vrai. Quand un agent exécute look $\left(s d s_{p}, s d s_{r}, e\right)$, l'ensemble d'objets de l'environnement $C$ est sélectionné pour appariement avec $e$ (chaque variable est unifiée avec un objet de $C$ ). L'expression $e$ doit être évaluée à vrai avec cette unification pour que look soit exécuté. Les objets associés avec les variables dans $s d s_{p}$ sont perçus et ceux associés avec les variables dans $s d s_{r}$ sont récupérés. Par exemple, l'instruction :

$$
\begin{gathered}
\operatorname{look}(\{\text { train } \leftarrow t r\},\{\text { ticket } \leftarrow \text { tk }\}, \text { tr.destination }=\text { "London" } \\
\wedge t k . \text { prix } \leq \text { budget } \wedge \text { tk.train }=\text { tr.id })
\end{gathered}
$$

cherche deux objets qui seront unifiés avec $t k$ et $t r$. L'agent recherche un train allant à Londres et un ticket à bord de ce train avec un prix qu'il peut se permettre vu son budget. L'objet associé avec $t r$ (l'état publique du train) va être perçu tandis que l'objet associé avec $t k$ (le ticket) va être récupéré. Après l'exécution de cette instruction, les deux objets vont être présents dans la mémoire locale de l'agent appelant, qui va avoir deux propriétés additionnelles de type référence : ticket, qui se réfère à l'objet associé avec la variable $t k$ et train, qui se réfère à l'objet associé avec $t r$. L'objet unifié avec $t k$ (représentant le ticket) ne sera plus présent dans $\Omega_{E N V}$. L'appariement dans LACIOS matérialise ce que nous appelons interaction contextuelle, qui est le type d'interaction qui utilise l'état de l'agent et l'état des objets dans l'environnement pour accéder à un ensemble d'objets. A titre de comparaison, les templates de Linda permettent de conditionner l'accès à une données par son seul état propre.

\subsection{Interaction avec des systèmes/utilisateurs externes}

Le langage de coordination défini jusqu'à présent spécifie un SMA ouvert dans le sens où les agents peuvent rejoindre le système - avec spawn - et découvrir les autres agents et les données échangées - avec look - mais il s'agit d'un SMA clos dans le sens où on ne peut exprimer son interaction avec un système externe. En effet, toutes les données nécessaires à l'exécution d'un programme doivent exister dans le code des agents. Par exemple, un agent voyageur représente un usager humain dans le SMA, dont les propriétés (destination, budget, etc.) sont inconnues avant l'exécution. Ici, les valeurs des propriétés d'un agent voyageur proviennent d'un système externe (serveur Web par exemple). Nous étendons la notion de variable afin d'exprimer l'action d'un système externe sur le comportement d'un agent. Voici l'instruction lançant un agent voyageur ayant des propriétés qui seront connues lors de l'exécution grâce à l'action d'un système externe :

$$
\operatorname{spawn}(P,\{\text { budget } \leftarrow ? b, \text { age } \leftarrow ? a, \text { fumeur } \leftarrow ? f \text {, destination } \leftarrow ? d\})
$$

avec $? a, ? b, ? d$ et $? f$ des variables. Lors de l'exécution, le système externe affecte des valeurs à ces variables. Le système externe n'est pas modélisé, seule son action est observée. 
La syntaxe d'une expression est augmentée avec les variables ainsi :

\begin{tabular}{l}
\hline$e::=\ldots$ \\
$\mid ? x \quad$ avec $? x \in \mathcal{X}$ \\
\hline
\end{tabular}

Les variables d'une expression prennent une valeur d'une manière non déterministe dans leur domaine de valeurs (leur type).

L'introduction des variables pour l'interaction avec un système externe est intéressante dans la mesure où elle sépare clairement l'aspect coordination - ce que fait le programme - et l'aspect interaction avec un système externe, i.e. la manière avec laquelle il est utilisé. Ainsi, dans le processus $P=\operatorname{spawn}(P,\{$ budget $\leftarrow$ ?b, age $\leftarrow$ ?a, fumeur $\leftarrow$ ?f, destination $\leftarrow$ ? $d\}$ ), peu importe le système instanciant les variables de $P$ (serveur Web, interface graphique, simulateur, etc.) le code relatif au comportement des agents reste le même. Afin de demander l'instanciation d'une variable, un agent utilise un opérateur spécifique $\nu . \nu X$, avec $X$ un ensemble de variables, a pour effet de donner, d'une manière non déterministe, une valeur dans leur domaine de définition à toutes les variables dans $X$.

\section{La gestion de la sécurité}

Nous décidons de garder un partage total des données entre tous les agents dans l'espace des données, et non de les cloisonner dans des environnements privés (de type tuplespaces multiples). Nous restons par ce fait fidèles au modèle Linda initial, et rencontrons donc les mêmes problèmes de sécurité. Plus précisément, des insertions frauduleuses de données peuvent se produire et ni les agents ni le concepteur ne peuvent les empêcher. Dans LaCios, les agents sont responsables des objets qu'ils mettent dans l'environnement. Pour éviter l'utilisation frauduleuse de ces objets, le langage supporte deux niveaux de contrôle, un niveau global pour le concepteur pour contrôler l'insertion d'objets et un niveau local pour les propriétaires des objets (les agents) pour contrôler la manière avec laquelle leurs objets vont être utilisés. Le niveau global gère chaque insertion d'objets dans l'environnement. Il vérifie si les propriétés de l'agent lui donnent le droit d'insérer l'objet. Cette vérification garantirait notamment l'authenticité des messages émis, en vérifiant que l'agent n'est pas en train de se faire passer pour quelqu'un d'autre. Le niveau local garde chaque perception et récupération d'objets. Il vérifie que le propriétaire de l'objet permet bien sa perception ou sa récupération par l'agent qui en fait la demande. Ces vérifications garantissent la confidentialité, la disponibilité et l'intégrité des données échangées. Il est notable que LACIOS gère la sécurité suivant le modèle originel de Linda, i.e. associativement. La communication demeure anonyme et la sécurité est gérée symboliquement, tout en maintenant un partage total des données entre les agents du système. 


\subsection{Contrôle global}

Le concepteur du middleware est le plus à même de définir les conditions spécifiant que certaines insertions d'objets sont frauduleuses. Nous lui fournissons donc un contrôle global des insertions d'objets par les agents. Une menace à l'authenticité (quand un agent essaie de se faire passer pour quelqu'un d'autre par exemple) est une illustration d'une insertion frauduleuse. Plus généralement, les objets ajoutés dans l'environnement pourraient corrompre la cohérence des données suivant la logique de l'application (résultant en deux agents avec la même position, ou avec une nouvelle enchère inférieure à l'enchère courante, etc.).

Considérons par exemple l'action suivante :

add $\left(\left\{\right.\right.$ from $\leftarrow$ compagnon $_{2} . i d$, to $\leftarrow$ compagnon $_{1} . i d$, subject $\leftarrow$ "coalition" $\left.\}\right)$

Cette action est frauduleuse, puisque l'agent essaie d'envoyer un message à son premier compagnon avec un $i d$ différent du sien $(d($ from $) \neq i d)$.

Cette première classe de menaces (qui incluent la menace à l'authenticité) concerne les règles de sécurité qui doivent être vérifiées quand un add est exécuté. Pour gérer ces menaces résultant de l'insertion frauduleuse d'objets dans l'environnement, le concepteur identifie les situations critiques et spécifie chacune en définissant une règle de sécurité $s(s \in \mathcal{S}, \mathcal{S} \subseteq \operatorname{Exp}$ est l'ensemble des règles de sécurité du système). Une expression $s$ dans $\mathcal{S}$ est une expression booléenne dans laquelle le concepteur spécifie les conditions sur l'état de l'agent exécutant add et les conditions sur la description de l'objet qu'il ajoute. Pour ce faire, nous ajoutons un mot clé spécifique that dans la syntaxe d'une expression pour désigner, dans une règle de sécurité, l'objet ajouté par l'agent.

$$
e::=\cdots \mid \text { that.e }
$$

Par exemple, voici l'expression empêchant un agent d'insérer un objet qui a une propriété from qui est différente de la sienne : $s \equiv$ that.from $=i d$, où $i d$ désigne l'identifiant de l'agent exécutant add et that désigne l'objet ajouté par l'agent. Quand un agent a exécute add $\left(\left\{\right.\right.$ from $\leftarrow$ companion $_{2} . i d$, to $\leftarrow$ companion $_{1} . i d$, subject $\leftarrow$ "coalition" $\left.\}\right)$, la règle de sécurité specifiée par le concepteur est évaluée à $f a u x, \operatorname{car} d($ from $) \neq d_{a}(i d)$, et l'opération est annulée.

La vérification et la maintenance de la cohérence de l'ensemble des règles globales de sécurité est en dehors du périmètre de cet article. 


\subsection{Contrôle local}

Les agents du système sont les plus à même de connaître les conditions faisant que la perception ou la récupération d'un objet qu'ils insèrent soit frauduleuse. Nous leur fournissons donc un contrôle local pour gérer l'observabilité de leurs propres objets. Une menace à la confidentialité (e.g. l'interception par un agent d'informations ou de messages confidentiels d'un autre), ou une menace à la disponibilité (e.g. la suppression par un agent des informations ou des messages d'un autre) sont des exemples de tels accès frauduleux. Nous proposons de permettre aux agents de définir les règles d'observabilité - sur la perception et sur la récupération - et de laisser l'environnement vérifier que ces conditions sont respectées.

Ceci est fait en permettant à un agent, quand il insère un objet, de gérer son observabilité, i.e. d'identifier les situations où la perception ou la récupération de l'objet inséré est interdite. Pour ce faire, la syntaxe de la primitive add est complétée comme suit.

$$
\mu::=\cdots \mid \operatorname{add}\left(s d s, e_{p}, e_{r}\right)
$$

où $e_{p}$ et $e_{r}$ sont des expressions booléennes. L'expression $e_{p}$ spécifie les conditions qu'un agent doit satisfaire pour avoir le droit de percevoir l'objet décrit par $s d s$, et $e_{r}$ définit les conditions qu'un agent doit satisfaire pour avoir le droit de le récupérer. Quand un agent exécute $\operatorname{look}\left(s d s_{p}, s d s_{r}, e\right)$, pour chaque objet $o \in C$ (l'ensemble des objets sélectionnés pour appariement depuis l'environnement) qui est unifié avec une variable dans $s d s_{p}$, l'expression $e_{p}$ associée à $o$ doit être évaluée à vrai, et pour chaque objet $o$ unifié avec une variable dans $s d s_{r}$, l'expression $e_{r}$ associée avec $o$ doit être évaluée à vrai. Sinon, l'action look ne peut pas être exécutée avec cet ensemble d'objets. Quand l'agent ne veut pas restreindre la perception ou la récupération de l'objet décrit par $s d s$, il affecte vrai à $e_{p}$ ou $e_{r}$, respectivement. Par exemple, soit l'agent $a$ (supposons que l'id du compagnon de $a=5$ ) qui veut éviter que le message qu'il a adressé à son compagnon ne soit récupéré par d'autres, et d'être perçu par des agents autres que lui-même (le mot clé that a ici la même sémantique, i.e. il désigne l'objet inséré $):$ add $(\{$ from $\leftarrow i d$, to $\leftarrow$ compagnon.id, subject $\leftarrow$ "coalition" $\}, i d=$ that.from, $i d=$ that.to)

Considérons un agent $b$ avec $d_{b}(i d)=10$ qui exécute

$\operatorname{look}(\{$ receiver $\leftarrow r\},\{$ message $\leftarrow m\}, m . t o=r . i d \wedge$ r.destination $=$ destination $)$

L'agent $b$ est en train d'essayer de récupérer un message (objet unifié avec $m$ ) et de percevoir l'objet représentant l'agent auquel $m$ est adressé (objet unifié avec $r$ ), si sa destination est égale à sa propre destination. Grâce aux conditions associées avec l'objet inséré, $b$ ne sera pas capable de percevoir le message de $a$. Concrètement, tout appariement essayant d'unifier $m$ avec le message de $a$ est interdit par l'environnement et n'est pas considéré. 
Mise à part l'authenticité, la confidentialité et la disponibilité, une quatrième menace à la sécurité des informations est l'intégrité. Les menaces à l'intégrité sont relatives à la modification par un agent des messages ou des informations d'un autre. Puisque la primitive update est locale dans LACIOS, aucun agent ne peut modifier les informations des autres. La seule manière pour ce faire - comme c'est le cas pour tous les langages orientés données - est de récupérer un objet et d'en créer un nouveau avec des informations différentes. La récupération d'objets est déjà gardée par les règles de sécurité locales anti-interception. Par conséquent, il n’y a nul besoin d'un mécanisme spécifique contre les menaces à l'intégrité dans LACIOS.

Une cinquième menace à la sécurité des informations est généralement citée, qui est celle de non-répudiation, impliquant qu'un participant à une transaction ne peut nier avoir reçu une transaction, comme l'autre participant ne peut nier l'avoir envoyée. A cause de la communication générative de Linda, nous ne pouvons établir une correspondance entre un objet particulier et son émetteur ou récepteur. D'ailleurs, aucun des langages de coordination ne traite ce problème. Néanmoins, lors de l'implémentation, un système adhérant à notre modèle peut utiliser un log avec une telle correspondance, qui peut être lue par les agents, pour résoudre ce problème. Etant donné que l'authenticité est garantie avec notre mécanisme de contrôle global, le contenu de ce log est certifié correct.

Notons que, dans le développement de la gestion de la sécurité définie ci-avant, nous prenons seulement en compte la sécurité entre les agents locaux et l'environnement. Ce faisant, nous faisons deux suppositions. D'une part, un spawn d'un agent représentant un système externe, un utilisateur ou un agent doit être réalisé suivant un protocole de sécurité externe (comme décrit dans la sous-section 2.1) pour être certain qu'il s'agit bien de l'agent avec l'identifiant revendiqué. D'autre part, nous interdisons aux agents locaux d'essayer de changer leurs identifiants avec un update tout le long de l'exécution du système. Nous imposons cette interdiction en définissant une seule valeur dans le domaine de définition de la propriété concernée ; par conséquent, changer cette propriété provoquerait une erreur sémantique.

\subsection{Spécification du comportement des agents}

Ce paragraphe fournit la définition complète d'un SMA ouvert écrit en LACIOS, en commençant par la définition complète des primitives du langage.

$$
\mu::=\operatorname{add}(s d s)\left|\operatorname{look}\left(s d s_{p}, s d s_{r}, e\right)\right| \text { update }(s d s) \mid \operatorname{spawn}(P, s d s)
$$

Nous sommes à présent prêts à définir les processus, qui définissent le comportement des agents. La primitive spawn $(P, s d s)$ lance un nouvel agent qui se comporte comme le processus $P$ et dont la description est le résultat de l'évaluation de $s d s$ (sa transformation en une description $d s$ ). Ci-après la définition complète d'un processus. 
Définition 10 (Processus) Étant donné un ensemble d'identifiants de processus $\left\{K_{i}\right\}_{i \in I}$, une définition de processus est sous la forme $: \forall i \in I, K_{i} \stackrel{\text { def }}{=} P_{i}$, où chaque $P_{i}$ est généré via la grammaire du tableau 2.

\begin{tabular}{ll}
\hline$P::=\mathbf{0}$ & (processus nul) \\
$\mid \mu . P$ & (préfixage par une action) \\
$\mid b\lfloor P\rfloor+b\lfloor P\rfloor$, où $b$ est une expression booléenne & (choix) \\
$\mid P \| P$ & (composition parallèle) \\
$\mid \nu X(P)$ & (liaison de variables) \\
$\mid K_{j}$, pour un certain $j \in I$ & (invocation de processus) \\
$\mu::=\operatorname{spawn}(P, s d s)\left|a d d\left(s d s, e_{p}, e_{r}\right)\right| \operatorname{loo}\left(d c_{p}, d c_{r}, e\right)$ & $\mid$ update $(s d s)$ \\
$\begin{array}{ll}\text { avec } e_{p} \text { et } e_{r} \text { des expressions, } s d s \text { une description symbolique } \\
d c_{p} \text { et } d c_{r} \text { des descriptions de contexte }\end{array}$ & \\
\hline
\end{tabular}

Tableau 2. Syntaxe d'un Processus

Les processus, que nous dénotons $P, Q, \ldots$ représentent les programmes du système, et les comportements des agents qui y évoluent. Un programme peut être terminé $\mathbf{0}$ (habituellement omis). Il peut être une expression de choix entre programmes $b\lfloor P\rfloor+b\lfloor P\rfloor$, où chaque $P$ est gardé par l'évaluation d'une expression booléenne $b$ : quand $b$ est évaluée à vrai, le programme $P$ est exécuté. Avec cette syntaxe, nous pouvons exprimer aussi bien un choix déterministe qu'un choix non déterministe. En effet, $b\lfloor P\rfloor+\neg b\lfloor Q\rfloor$ est un choix déterministe entre $P$ et $Q$, alors que vrai $\lfloor P\rfloor+$ vrai $\lfloor Q\rfloor$ est un choix non déterministe. La syntaxe du choix peut se généraliser à plus de deux processus. En effet, l'expression qui suit est un choix entre trois processus $P, Q$ et $R: b_{1}\lfloor P\rfloor+\left(b_{2} \vee b_{3}\right)\left\lfloor b_{2}\lfloor Q\rfloor+b_{3}\lfloor Q\rfloor\right\rfloor$. Un programme peut aussi être une composition parallèle de programmes $P \| Q, P$ et $Q$ sont donc exécutés en parallèle. Un programme peut être une invocation d'un autre processus ayant comme identifiant la constante $K_{j}$, et se comporte comme le processus défini par $K_{j} . \nu X(P)$ se comporte comme $P$ où toutes les variables libres dans l'ensemble $X$ sont liées avec des valeurs dans leur type. Un programme peut également être un processus préfixé par une action $\mu . P$. Les actions sont les primitives du langage, comme présentées plus tôt.

Nous sommes à présent capables de présenter la structure du SMA coordonné, illustré par la figure 3. Dans ce schéma, les objets sont représentés par l'ensemble $\mathcal{O}$, et les agents par l'ensemble $\mathcal{A}$. Chaque agent $a$ a une mémoire locale $\Omega_{a}$, dans laquelle sont stockés les objets perçus et reçus. Les objets dans $\Omega_{E N V}$ et dans $\Omega_{a}$ sont considérés comme des références vers des objets dans $\mathcal{O}$. Un agent a accès à sa propre description (quand $\pi$ est rencontrée dans le code d'un agent $a$, elle se réfère à $d_{a}(\pi)$ ) 
et est défini également par un comportement ( $P$ et $Q$ dans la figure). L'environnement est quant à lui constitué d'un ensemble de références vers des objets dans $\mathcal{O}$.

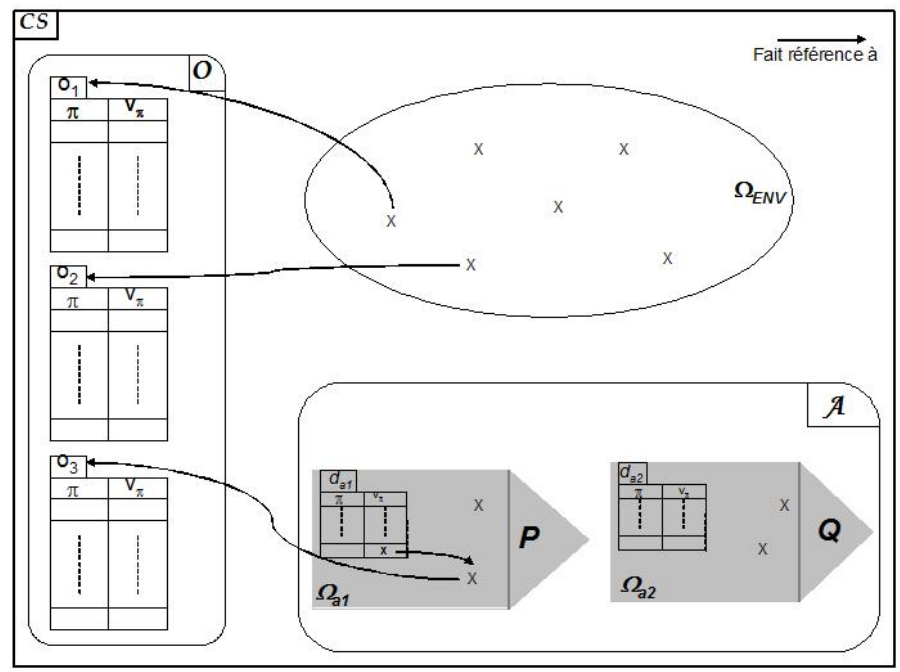

Figure 3. SMA Coordonné

L'état d'un SMA coordonné est défini comme suit.

Définition 11 (SMA Coordonné) $C S=\left\langle\Omega, d, \Omega_{E N V}, \mathcal{S}\right\rangle$,

$-\Omega=\mathcal{A} \uplus \mathcal{O}$ est l'ensemble d'entités, composé de $\mathcal{A}$ l'ensemble des agents et $\mathcal{O}$ l'ensemble des objets,

$-\mathcal{A} \subseteq \Omega$ est l'ensemble d'agents.

- $\Omega_{a}$ est la mémoire privée de l'agent $a, \Omega_{a} \subseteq \mathcal{O} \cup\{a\}$, i.e. l'agent a accès à sa propre description,

- proc(a) est le processus décrivant le comportement de l'agent a.

$-\mathcal{O} \subseteq \Omega$ est l'ensemble d'objets.

- $e_{p}(o)$ renvoie le prédicat spécifiant les conditions de perception de l'objet o, i.e. quels agents peuvent percevoir $o$.

- $e_{r}(o)$ renvoie le prédicat spécifiant les conditions de réception de l'objet $o$,

$d: \Omega \rightarrow(\mathcal{N} \rightarrow \mathcal{T})$ est la fonction de description du SMA, chaque $d(\omega)$ est une description d'entité comme décrite précédemment (dénotée également par $d_{\omega}$ ),

$\Omega_{E N V} \subseteq \mathcal{O}$ est l'ensemble d' objets qui sont dans l'environnement, 
$\mathcal{S} \subset$ Exp est l'ensemble des prédicats spécifiant les conditions devant être vérifiées, lors de l'exécution d'un add, par l'agent appelant et par la description ajoutée, afin d'être validé.

La mémoire locale d'un agent est composée des objets qu'il a perçus et reçus. L'environnement est $\Omega_{E N V}$, tous les objets ajoutés par des add y sont placées.

\section{Le langage de programmation J AVA-L ACIOS}

Nous avons proposé un langage de coordination indépendant de tout langage de programmation particulier. La procédure habituelle d'implémentation d'un langage de coordination est de fournir des librairies dans un langage de programmation hôte qui peuvent être utilisées par d'autres programmes qui désirent être coordonnés selon le modèle (e.g. Klava associé à Klaim (De Nicola et al., 1998)). Cependant, cette solution ne tire pas un profit maximal de la sémantique opérationnelle associée au langage. En effet, c'est le programmeur qui doit lui-même veiller au respect de cette sémantique dans chaque système qu'il réalise. Nous avons donc choisi de concevoir un outil permettant d'écrire un programme dans la syntaxe de LACiOS, et de générer un système prêt à être utilisé. L'avantage pour le programmeur est d'avoir la garantie que son programme adhère bien au modèle de coordination. Notamment, nous désirons utiliser les opérateurs de préfixage, de choix, de composition parallèle et de liaison des variables libres comme décrits par la syntaxe, dans la définition du comportement des agents. De plus, en nous fondant sur la syntaxe et la sémantique de LACIOS, nous pourrions réaliser des compilateurs pour LACIOS vers tout autre langage cible.

Pour illustrer une implémentation particulière de LACIOS, notre choix du langage de programmation cible s'est porté sur Java. Ce choix est motivé par la simplicité de création et de gestion de processus concurrents (les Threads), ainsi que la facilité de création de parseurs, grâce au générateur de parseurs Java JavaCC ${ }^{1}$.

Un programme LACiOS est un fichier texte où sont décrits les comportements des agents ainsi que le système coordonné. Un système coordonné est défini par l'ensemble des agents initiaux, lancés au début de l'exécution, ainsi que les règles de sécurité $\mathcal{S}$. Les programmeurs rédigent les scripts LACIOS décrivant le comportement de leurs agents. Les scripts sont parsés et compilés, générant un objet programme Java. Lors de l'exécution, une application externe peut manipuler l'objet programme et éventuellement lier les variables libres des agents le composant.

Afin de simplifier le travail du programmeur, notre outil permet de visualiser les composantes du programme avant le démarrage et en cours d'exécution. Avant l'exécution, le programmeur peut visualiser le graphe de transition entre états d'un agent (la figure 4 illustre l'exécution de notre outil pour une application réelle de transport (Zargayouna et al., 2009)). Chaque état représente une action de l'agent et les

1. http ://javacc.dev.java.net/ 


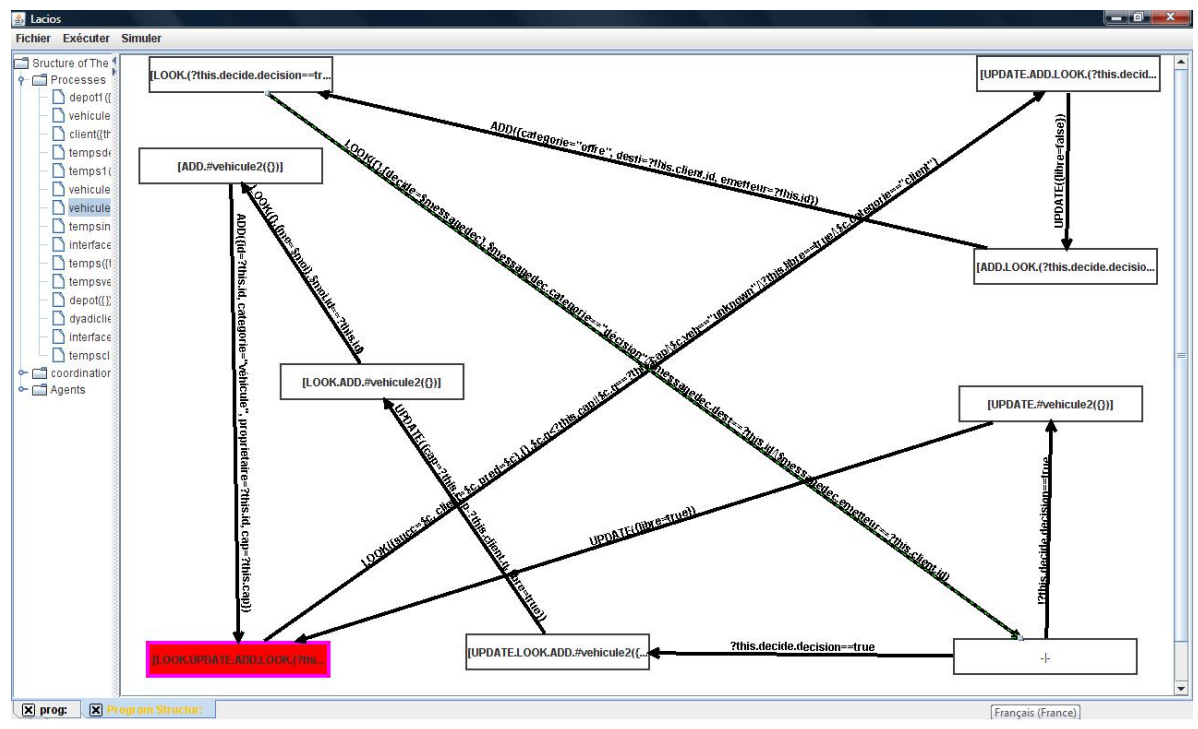

Figure 4. Système de Transitions Étiquetées d'un agent LACios

arcs représentent l'exécution de cette action par l'agent (dans le cas d'un add d'un update, ou d'un spawn) ou de l'environnement (dans le cas d'un look). En cours d'exécution, notre outil propose une seconde interface graphique permettant à l'utilisateur de visualiser l'état du programme. Il peut ainsi vérifier le bon déroulement et inspecter en temps réel les objets présents dans l'environnement ainsi que les comportements des agents en cours d'exécution. Un agent est un objet Java ayant une description et un comportement. Le comportement d'un agent est fait d'une composition de Threads Java. Une seule instance d'objet de la classe «Environnement » est définie dans un programme JAVA-LACIOS, et tous les agents du système ont une référence vers celle-ci. Un environnement est défini par l'ensemble d'objets qu'il contient, une liste d'expressions en attente (bloquées sur des look qui ne trouvent pas d'appariement valide), et un ensemble de règles de sécurité $\mathcal{S}$ (expressions). L'environnement a deux méthodes : look et $a d d$, représentant les primitives du langage responsables de l'interaction avec l'environnement.

La traduction d'un programme LACiOs en java réalisé par JavaCC est directe. Cependant, il a été nécessaire de prendre en compte des spécificités propres à Java. En effet, l'accès concurrent aux objets de l'environnement nécessite une synchronisation des exécutions de add et look. Or, suivant le modèle originel Linda, l'instruction add ne doit pas bloquer un agent tant que l'environnement n'a pas rendu la main. Afin de résoudre cette contradiction, nous avons défini un espace tampon où les agents déposent leurs objets sans être bloqués et c'est ce tampon qui est synchronisé avec l'environnement pour l'exécution de l'instruction look. D'autre part, afin d'assurer que tout agent accède à un environnement cohérent, éviter par exemple qu'il perçoive des 
objets qui ont été reçus par un autre agent (donc absent de l'environnement), l'environnement est bloqué lors d'un appel de l'instruction look tant que l'appariement correspondant n'est pas terminé. Si l'appariement échoue, le processus de l'agent ayant fait l'appel est bloqué et ne sera pris en compte que si de nouveaux objets sont ajoutés à l'environnement.

Enfin, l'instruction update modifie «localement» les propriétés d'un agent mais influence son interaction avec l'environnement. En effet, si une instruction look est en cours d'exécution pour un agent dont les propriétés ont été mise à jour par une instruction update, le résultat de l'appariement dépend de ces nouvelles valeurs. Pour ce même agent, toutes les instructions look prenant en compte les propriétés mises à jour et dont l'appariement a échoué sont à nouveau évaluées.

L'intérêt scientifique du développement de JAVA-LACIOS a été pour nous de deux ordres. D'une part, cela nous a permis de vérifier que la sémantique opérationnelle proposée est assez précise pour une implémentation de LACIOS dans un langage hôte. D'autre part, cela nous a permis de réaliser et d'effectuer des expérimentations avec un système multi-agent de transport à la demande écrit en JAVA-LACIOS (Zargayouna, 2007).

Une implémentation de LACIOS dans l'un des langages supportant la concurrence et la répartition (e.g. Erlang (Armstrong et al., 1996)) est également possible. Pour ce faire, l'utilisation de bases de données Mnesia (Nilsson et al., 1996) pour simuler l'interaction associative à la Linda nous semble une piste prometteuse.

\section{Travaux connexes}

Le travail que nous décrivons dans ce papier a comme objectif premier d'améliorer la sécurité dans les langages orientés données pour le développement de SMA ouverts. Nous voulions équiper ces langages avec un mécanisme de sécurité grains-fins fondé sur les états des agents. La poursuite de cet objectif nous a amené à développer un nouveau langage, LACIOS, qui offre ces caractéristiques. Nous avons successivement ajouté une nouvelle structure de données, décrit des agents avec états, proposé un nouveau mécanisme d'appariement, ouvrant ainsi la voie au mécanisme de sécurité que nous adoptons. Par rapport à la classification des langages orientés données sécurisés de (Focardi et al., 2006), notre proposition est une approche orientée entités, qui associe des informations additionnelles aux ressources, listant les agents qui peuvent y accéder. L'originalité de notre approche par rapport à ces propositions est que, au lieu de lister les agents qui peuvent accéder à une donnée, les agents sont décrits «symboliquement», i.e. leurs propriétés sont définies sans les pointer nommément. Nous laissons les agents spécifier, quand ils insèrent une donnée dans l'environnement, les conditions sous lesquelles elle peut être lue ou récupérée par les autres. Nous laissons également le concepteur spécifier les conditions pour qu'un agent puisse ou ne puisse pas insérer une certaine donnée à l'espace, suivant la logique de l'application. 
Généralement, la sécurité dans les langages de coordination orientée données est mise en oeuvre en utilisant des espaces (logiques) multiples, en définissant des « lois d'interaction» ou en définissant des rôles et des droits d'accès associés à eux. Avec les espaces multiples (e.g. Klaim (De Nicola et al., 1998), SecSpaces (Busi et al., 2003a; Bravetti et al., 2004), SecOS (Vitek et al., 2003), etc.), deux agents qui souhaitent échanger des données confidentielles utilisent un espace qu'ils sont les seuls à connaître. Cependant, quand la sécurité est garantie en isolant les données dans des espaces privés, accéder à un espace donne la possibilité d'accéder à toutes ses données, et en être exclu signifie n'avoir accès à aucune de ses données. Dans LACIOS, les agents ont un état, et un agent peut protéger ses données finement (chaque objet est protégé individuellement) sans connaître les autres agents, ce qui permet d'avoir une interaction sécurisée avec un partage complet des données. Les rôles et les droits d'accès (comme dans le modèle RBAC associé à TuCSon (Omicini et al., 2005)) sont une couche supplémentaire au dessus des espaces multiples. Par conséquent, la sécurité est également définie à gros-grain. Tagged Sets (Oriol et al., 2005) permet une protection fine des données ajoutées dans l'espace de données. Cependant, ni les états des agents, ni des opérateurs de comparaison ne sont définis comme c'est le cas dans LACIOS. Les « lois d'interaction» dans LGI (Minsky et al., 2001) permettent aux données d'être sécurisées en spécifiant des conditions sur les états des agents et sur le contenu des données. Des réactions du tuplespace sont associées avec les actions des agents de telle manière qu'elles résultent toujours en une configuration cohérente. Néanmoins, deux points différencient LACIOS de LGI. D'une part, les lois dans LGI sont définies par le concepteur seulement, alors que les agents ne le peuvent pas. D'autre part, les lois sont des règles actives, ce qui pose le problème de non-terminaison du processus d'appariement (une action suivie d'une chaîne infinie de réactions). Dans LaCios, les règles annulent des perceptions ou des récupérations mais ne lancent pas de réaction, le problème de non-terminaison ne se pose donc pas.

Dans (Busi et al., 2003a), des champs cryptographiques spécifiques sont ajoutés aux n-uplets pour authentifier le producteur d'une donnée et d'identifier les lecteurs/récupérateurs de cette donnée. Cette authentification est prise en charge dans LACIOS grâce aux états des agents et aux règles de sécurité, mais il est néanmoins toujours possible de définir une propriété spécifique pour les champs cryptographiques. Finalement, dans (Focardi et al., 2006), les auteurs signalent que « la version sécurisée de Lime (Murphy et al., 2006) est la seule qui permet de contrôler les opérations d'output, et SecSpaces (Busi et al., 2003a) est le seul qui permet de distinguer entre les processus qui peuvent consommer et ceux qui peuvent lire un certain n-uplet». LACIOS permet en même temps de contrôler les insertions et de distinguer entre la lecture d'une donnée et sa récupération.

\section{Conclusion et perspectives}

L'investigation des enjeux de sécurité dans les langages de coordination orientés données nous a amené à proposer un langage modifié permettant une protection 
grain fin des données échangées via l'espace de données partagées. Ce papier définit LACIOS, qui peut être utilisé dans la modélisation d'un grand nombre d'applications dans lesquelles les agents rejoignent et quittent le système librement, où les agents interagissent avec des systèmes externes, et où la sécurité est cruciale. LACiOs participe à l'effort de recherche en cours sur la définition d'architectures, de modèles et de langages pour les SMA ouverts (e.g. TuCSon (Omicini et al., 1999), le framework OAA (Cheyer et al., 2001), etc.). Utiliser LACIOS facilite pour les concepteurs de SMA ouverts la traduction des concepts abstraits manipulés par les agents et leur besoins en interaction à des constructeurs syntaxiques, tout en garantissant la sécurité des informations et exprimant des contraintes complexes. Relativement aux langages multi-agents existants, LACIOS permet de tirer avantage du modèle d'espace partagé au lieu de la communication à base de canaux utilisé dans la majorité des approches sécurisées. Nous avons démontré cette utilité pour une application complexe de transport dans un article récent (Zargayouna et al., 2009). L'introduction des états des agents, du modèle de données à base de couples propriété-valeurs, avec les opérateurs et les variables nous a conduit à proposer un nouveau langage au lieu de bâtir au dessus des langages existants. La sémantique opérationnelle de LACIOS peut être trouvée dans (Zargayouna, 2007).

Nous envisageons diverses directions pour nos travaux futurs. En amont de la définition du langage détaillé ici, nous prévoyons de définir un planificateur par agent dont le résultat serait les instructions impératives spécifiées dans LACIOS. En aval, nous prévoyons un mécanisme de distribution automatique de l'environnement fondé sur les treillis de Galois (regroupements d'agents et d'objets par similarité de propriétés).

Nos travaux futurs incluent également la considération de propriétés cryptographiques spécifiques pour assurer l'authenticité. Nous menons également un travail considérant l'ajout de constructeurs temporels dans LACIOS, inspiré par les travaux de (Busi et al., 2003b) et de (Linden et al., 2006). Notre objectif est d'exprimer l'insertion d'objets temporaires et pour définir une date butoir pour look avant qu'elle ne termine sans effet sur l'espace de données. La prochaine étape est d'utiliser ces nouveaux constructeurs dans la sécurité locale et globale pour permettre un mécanisme de sécurité plus riche permettant, par exemple, de détecter des utilisations multiples d'une carte de crédit dans un court laps de temps.

Les développements futurs de LACios comportent aussi la définition d'un mécanisme de contrôle global sur la primitive look. En effet, il pourrait s'avérer utile d'interdire aux agents de «tricher », en fournissant par exemple des informations qu'ils ne sont pas supposés communiquer aux autres. De plus, nous désirons améliorer le mécanisme de sécurité en permettant une « sécurité contextuelle globale », permettant de protéger les objets, selon la présence ou l'absence d'autres dans l'environnement. Par exemple, il permettrait de ne percevoir un objet que si l'agent l'ayant créé évolue toujours dans le SMA. Nous croyons que, grâce à l'interaction contextuelle, LACIOS est le langage le plus proche de la réalisation de ce type de sécurité, non considéré par les langages de la littérature. 


\section{Bibliographie}

Armstrong J., Virding R., Wikström C., Williams M., Concurrent Programming in ERLANG, Prentice Hall, Hertfordshire, UK, UK, 1996.

Bravetti M., Busi N., Gorrieri R., Lucchi R., Zavattaro G., « Security Issues in the Tuple-Space Coordination Model », Formal Aspects in Security and Trust, p. 1-12, 2004.

Bryce C., Cremonini M., « Coordination and security on the Internet », in A. Omicini, F. Zambonelli, M. Klusch, R. Tolksdorf (eds), Coordination of Internet agents : models, technologies, and applications, Springer-Verlag, chapter 11, p. 274-298, 2001.

Busi N., Gorrieri R., Lucchi R., Zavattaro G., « SecSpaces : a Data-driven Coordination Model for Environments Open to Untrusted Agents », Electronic Notes on Theoretical Computer Science, 2003a.

Busi N., Zavattaro G., « Expired data collection in shared dataspaces », Theoretical Computer Science, vol. 298, n 3, p. 529-556, 2003 b.

Carriero N., Gelernter D., Leichter J., « Distributed data structures in Linda », POPL'86 : Proceedings of the 13th ACM Sigact-Sigplan symposium on Principles Of Programming Languages, ACM Press, New York, NY (USA), p. 236-242, 1986.

Cheyer A., Martin D., « The Open Agent Architecture », Journal of Autonomous Agents and Multi-Agent Systems, vol. 4, n 1, p. 143-148, March, 2001.

Ciancarini P., « Coordination languages for open systems design », Proceedings of the International Conference on Computer Languages (ICCL'90), IEEE Computer Society, New Orleans, LA (USA), p. 252-260, 1990.

De Nicola R., Ferrari G. L., Pugliese R., « Klaim : A Kernel Language for Agents Interaction and Mobility », IEEE Transactions on Software Engineering, vol. 24, n 5, p. 315-330, 1998.

Dimitrakos T., Martinelli F. (eds), Formal Aspects in Security and Trust : Second IFIP TC1 WG1.7 Workshop on Formal Aspects in Security and Trust (FAST), an event of the 18th IFIP World Computer Congress, August 22-27, 2004, Toulouse, France, Springer, 2005.

El Fallah-Seghrouchni A., Les modèles de coordination d'agents cognitifs, Hermès IC2 (information, commande, communication), p. 139-177, 2001.

Focardi R., Lucchi R., Zavattaro G., « Secure shared data-space coordination languages : A process algebraic survey », Sci. Comput. Program., vol. 63, n 1, p. 3-15, 2006.

Freeman E., Arnold K., Hupfer S., JavaSpaces : Principles, Patterns, and Practice, AddisonWesley Longman Ltd., Essex (UK), 1999. 304 pages.

Gelernter D., Carriero N., « Coordination languages and their significance », Communications of the ACM, vol. 35, $\mathrm{n}^{\circ}$ 2, p. 97-107, 1992.

Komorowski J., Pawlak Z., Polkowski L., Skowron A., « Rough sets : a tutorial », in S. K. Pal, A. Skowron (eds), Rough-Fuzzy Hybridization : A New Method for Decision Making, Springer-Verlag, Singapore, 1998.

Linden I., Jacquet J.-M., Bosschere K. D., Brogi A., « On the expressiveness of timed coordination models », Science of Computer Programming, vol. 61, n² 2, p. 152-187, 2006.

Milner R., Communication and Concurrency, Prentice-Hall, 1989. 272 pages.

Minsky N. H., Minsky Y., Ungureanu V., « Safe Tuplespace-based Coordination in Multiagent Systems », Applied Artificial Intelligence, vol. 15, n 1, p. 11-33, 2001. 
Murphy A. L., Picco G. P., Roman G.-C., « LIME : A coordination model and middleware supporting mobility of hosts and agents », ACM Trans. Softw. Eng. Methodol., vol. 15, n 3, p. 279-328, 2006.

Nilsson H., Wikström C., Ab E. T., « Mnesia - An Industrial DBMS with Transactions, Distribution and a Logical Query Language », International Symposium on Cooperative Database Systems for Advanced Applications. Kyoto Japan, 1996.

Omicini A., Ricci A., Viroli M., « RBAC for Organisation and Security in an Agent Coordination Infrastructure », ENTCS, vol. 128, n ${ }^{\circ}$, p. 65-85, 2005. Proceedings of the 2nd International Workshop on Security Issues in Coordination Models, Languages, and Systems (SecCo 2004).

Omicini A., Zambonelli F., « Coordination for Internet Application Development », Autonomous Agents and Multi-Agent Systems, vol. 2, n 3, p. 251-269, 1999.

Oriol M., Hicks M., « Tagged Sets : a Secure and Transparent Coordination Medium », in J.-M. Jacquet, G. P. Picco (eds), Proceedings of the International Conference on Coordination Models and Languages (COORDINATION), vol. 3454 of Lecture Notes in Computer Science, Springer-Verlag, p. 252-267, April, 2005.

Papadopoulos G. A., Arbab F., « Coordination models and languages », Advances in Computers, vol. 46, p. 55, 1998.

Pfleeger C. P., Pfleeger S. L., Security in Computing, Prentice Hall Professional Technical Reference, 2002.

Sycara K. P., « Multiagent Systems », AI Magazine, vol. 19, n² 2, p. 79-92, 1998.

Vitek J., Bryce C., Oriol M., « Coordinating processes with secure spaces », Sci. Comput. Program., vol. 46, n 1-2, p. 163-193, 2003.

Wooldridge M., Jennings N. R., « Intelligent Agents : Theory and Practice », Knowledge Engineering Review, vol. 10, n² 2, p. 115-152, 1995.

Wyckoff P., McLaughry S., Lehman T., Ford D., « TSpaces », IBM Systems Journal, vol. 37, $\mathrm{n}^{\circ} 3$, p. 454-474, 1998.

Zargayouna M., Modèle et langage de coordination pour les systèmes multi-agents ouverts. Application au problème du transport à la demande, $\mathrm{PhD}$ dissertation, University of ParisDauphine, Paris (France), 2007. In french.

Zargayouna M., Balbo F., Scemama G., « A Data-Oriented Coordination Language for Distributed Transportation Application », The third International KES Symposium on Agents and Multi-agent Systems Technologies and Applications (KES-AMSTA'09), vol. 5559 of Lecture Notes in Artificial Intelligence, Springer-Verlag, Uppsala (Sweden), p. 283-292, 2009. 